\title{
Changes in the quality of life in severely disabled people following provision of powered indoor/outdoor chairs
}

\author{
Davies $A^{1 \mathrm{a}}$, De Souza $\mathrm{LH}^{1 \mathrm{~b}}$, Frank AO ${ }^{2}$ \\ ${ }^{1 \mathrm{a}}$ Research Assistant \\ ${ }^{1 b}$ Professor of Rehabilitation \\ ${ }^{2}$ Consultant in Rehabilitation Medicine and Rheumatology \\ ${ }^{1}$ Centre for Research in Rehabilitation, Dept of Health Studies, Brunel University, \\ Osterley Campus, Isleworth, Middlesex, TW7 5DU, UK \\ Tel 0208891 0121; Fax 0208891 2030; email lorraine.desouza@brunel.ac.uk \\ ${ }^{2}$ Disablement Services Centre, Royal National Orthopaedic Hospital, Stanmore, HA7 \\ 4LP
}

Correspondence to Prof. De Souza, address as above. 


\title{
Changes in the quality of life in severely disabled people following provision of powered indoor/outdoor chairs
}

\author{
Abstract \\ Purpose: To determine the benefits for patients who received an Electric \\ Powered Indoor/outdoor Chair (EPIOC) and to quantify their perceived changes to \\ their quality of life.
}

Method: Community-based cohort study of all patients provided with an EPIOC over 4 months; and followed up about 3 months later in a community served by a regional wheelchair service in North West London (population about 3.1 million) using the EuroQol EQ-5D with visual analogue scales for each of the 5 dimensions of the EQ-5D.

Results: $\quad$ Sixty-four wheelchair users were assessed initially and 51 completed follow up. Chair users showed no significant improvement in health state as measured by the EQ-5D after EPIOC provision. The visual analogue scales (VASs) indicated that, although perceived overall health state, independence and social life did not appear to improve, the dimensions of mobility, quality of life and pain/discomfort improved significantly on provision of an EPIOC.

Conclusion: EPIOC users reported significant improvements in several important aspects of their lives; not just in mobility (as expected) but also in reduction of pain 
Powered wheelchairs and quality of life

and discomfort. The use of VASs provided a more holistic set of outcome measures that demonstrate quality of life benefits beyond that of health state alone. 


\section{Introduction}

Electric Indoor/Outdoor Powered Wheelchairs (EPIOCs) are only available in the UK for very severely disabled individuals ${ }^{1}$. In 1997, a service was set up for the introduction of EPIOC chairs in the North West London Region ${ }^{2}$. Initial assessments by our service ${ }^{2,3}$ and others ${ }^{4-6}$ have suggested that these chairs may improve the quality of life for those who use them (the users).

Few studies have been performed that have aimed to demonstrate the benefit to users of EPIOC provision. None to our knowledge have tested the assumption that quality of life is significantly improved after EPIOC provision. Consequently we have tested this assumption in our cohort using their self-assessed quality of life before and after provision.

Quality of life issues are recognised as being important for wheelchair services ${ }^{7}$, have been reported anecdotally ${ }^{5}$ and are considered to be important by the users.

Initial audit has indicated that EPIOC users are a heterogeneous group ${ }^{3}$. Generic quality of life measures are likely to be the most appropriate. However the SF36 has been reported as being problematic in a similar group of severely disabled individuals ${ }^{8}$ and the EuroQol EQ-5D (EQ-5D) ${ }^{9}$ has been found to lack discrimination in highly disabled populations ${ }^{10}$. However, other domains such as 'vitality' and 'bodily pain' showed no such effect. Thus there is a continued need to test other instruments in such populations ${ }^{8}$. 
The EQ-5D ${ }^{9}$ is a widely used quality of life measure. It is short and easy to administer

11 and is designed to be appropriate when used as part of a face-to-face interview. The instrument consists of five dimensions with three levels of response for each in the form of a statement, and a visual analogue scale (VAS) on which respondents are asked to indicate how good or bad their health state is on the day of response. The aim of this study is to assess the change in quality of life after provision of an EPIOC using the EQ-5D.

\section{Subjects}

All potential users $(n=72)$ who were assessed as suitable for EPIOC provision by the Regional EPIOC Service between November 1998 and February 1999 were approached to participate irrespective of age, sex or diagnosis. Initial contact was made by telephone. Three people could not be contacted, three people declined to participate, one person was not interviewed due to wheelchair delivery being brought forward and one person failed to keep the appointment. Thus 64 users entered the study (28 male, 36 female; mean age 52 sd 21 years, range 14-83). Their main diagnoses are given in table 1.

[Insert table 1 about here]

\section{Methods}

The EQ-5D was presented as part of a face-to-face interview as some users were unable to write. All users were offered the option of marking their own responses or 
Powered wheelchairs and quality of life

having them marked on their behalf according to their instructions whilst simultaneously viewing the process. In almost all cases this latter option was chosen.

Subjects completed the five dimensions of the EQ-5D, each with three levels of response, and a single VAS. The mobility dimension asks respondents to indicate their current mobility by ticking the most appropriate of three statements, 'I have no problems in walking about', 'I have some problems in walking about', or 'I am confined to bed'. In an EPIOC user population, any reference to 'walking' is inappropriate. Face validity is compromised. Thus the mobility dimension of the EQ5D was modified to present the response statements as 'I am able to walk a few steps', 'I am able to transfer only' or 'I am not able to walk at all'. Users were also asked to complete five additional VASs in a thermometer style. These were for mobility, independence, social life, quality of life, and pain/discomfort, the same dimensions as used by the EQ-5D.

The assessment, including the VASs, was completed in accordance with the instructions in the EuroQol Users Guide ${ }^{12}$. The assessor was independent of the EPIOC service and the assessments were not available to any of the service providers.

Initial and follow-up interviews were conducted by appointment within the user's home or residential college. This allowed the users to respond to questions in familiar and relaxing surroundings and ensured that the interview context remained consistent. Interviews were conducted over nine months, during which time 51 patients received their EPIOC. The mean time to follow up interview was 97 (sd 16) days. At the follow 
Powered wheelchairs and quality of life

up assessment, users were asked to provide ratings on the current VASs compared to their baseline ratings. Thus, a relative opinion was required.

\section{Methods of analysis}

The EQ-5D was scored using a table of pre-determined values from the user guide ${ }^{12}$. Visual analogue scores obtained prior to EPIOC delivery (Pre-EPIOC) were compared with those scores obtained from the same respondents at follow-up (Post-EPIOC). The Wilcoxon Matched-Pairs Signed Ranks test (two-tailed), with Bonferroni corrections, was used to determine significant differences between pre and post-EPIOC conditions. Data sets were analysed using SPSS 8.0 for Windows.

\section{Results}

Fifty-one people (table 1) received chairs during the study period and completed both assessments. The before and after mean scores of the EQ-5D were 0.098 (sd 0.25) and 0.119 (sd 0.29) respectively. As expected in a group of severely disabled subjects, no significant differences were found and no significant differences were found for any of the subsections either (data not shown).

EPIOC users were asked to rate their own health state using the VAS in the EQ-5D prior to and after EPIOC delivery. Two subjects were unable to conceptualise their condition using this method and therefore did not provide a response. The mean estimated health ratings for the 49 subjects were 56.9 (sd 21.9) at baseline and 55.7 (sd 24.2). No significant difference was found for the 'health' dimension. Neither were there significant findings for the VAS responses in the dimensions of social life and 
independence. However there were significant improvements for the VAS responses in the dimensions of 'pain/discomfort', 'mobility' and 'quality of life' (table 2).

[Insert table 2 about here]

\section{Discussion}

Lack of EPIOC service research

Recognising that the field of EPIOC provision in the UK has not been researched adequately ${ }^{3}$, the lack of data on patients' experiences is even more dramatic. Our searches have only revealed few publications incorporating EPIOC users' views on their experiences ${ }^{5,6}$. To our knowledge no previous study has attempted prospectively to assess changes in the quality of life after EPIOC provision. The Edinburgh study examined user handicap before and after provision, but limited the study to the use of instruments rather than eliciting user views ${ }^{4}$. The Department of Health evaluation performed by the University of York was a retrospective survey, although large numbers were reported ${ }^{6}$.

\section{Sample}

Although the study sample was modest, the range of diagnoses was not different proportionately to our previous study ${ }^{3}$, although the mean group age (53) was somewhat higher. At least a third had progressive conditions, e.g. multiple sclerosis and muscular dystrophy (table 1) and their health would be likely to deteriorate over time. Sampling from community registers might give a broader range of disability ${ }^{8,13}$. However, samples also appear to vary across centres for EPIOC provision ${ }^{14}$. Some of 
these users would have been previous EPIOC users, and would not be expected to have any improvement in their quality of life ${ }^{3}$.

\section{Quality of life}

The results from the adjusted EQ-5D for EPIOC users showed that the instrument may have limited value in detecting change in the perceived quality of life of such severely physically disabled people. It may also be that generic quality of life scales may have limited value in evaluating specific health service interventions ${ }^{15}$, such as EPIOCs. Our results showed that the EQ-5D was too blunt an instrument to detect improved quality of life in severely disabled wheelchair-users. A similar finding was observed with those with rheumatic disorders using the EQ-5D ${ }^{11}$ and with the SF36 ${ }^{8,16}$.

The VASs on the other hand supported findings previously reported anecdotally ${ }^{2,3}$. There has been a suggestion that VAS use is more helpful in identifying lesser changes than the broad EQ-5D categories as a VAS has non-restricted scaling ${ }^{11}$. Therefore, it appears that the VASs allow the user more freedom to report changes according to their own views.

Quality of life is considered to be a complex concept including not only health and disability dimensions, but also the wider social and psychological dimensions ${ }^{17}$. In order to maintain respondents' own perceptions, interpretation of the meaning of each dimension was not imposed, and respondents were free to interpret each in their own terms and they could respond using their own individual value system for "quality of life'. Therefore users self-assessment using visual analogue scales could have provided 
Powered wheelchairs and quality of life

the opportunity for a more holistic assessment of this complex concept on an individual basis. This contrasts with the pre-defined dimensions of the EQ-5D, each with a specific field of response. Within the EQ-5D user guide ${ }^{12}$ the problem of limited statements is recognised.

There is no evidence to suggest that a person may not be both 'healthy' and disabled simultaneously. A small improvement of mean health state for recipients was suggested after receiving an EPIOC when the EQ-5D was used. This was unexpected in view of the progressive nature of some users' conditions.

Although the level of ability to perform activities may be unaffected by the introduction of an EPIOC, the range of activities may change following this intervention. Therefore the ability to perform new activities after intervention is a greater indication of benefit than performance levels on existing activities. This aspect of improvement will not be elicited by the EQ-5D, but could well enter the more holistic self-assessment obtained by the VASs.

The finding that pain and discomfort was significantly reduced after EPIOC provision is important. A previous survey has reported that $26 \%$ of EPIOC users complain of discomfort ${ }^{3}$ but when followed up 1.9 years after provision this figure had risen to $46 \%{ }^{18}$. This dimension of pain and discomfort posed problems in that people experienced difficulty in commenting on levels of pain or discomfort in the categories of 'none', 'moderate' and 'extreme'. Pain intensity is known to be difficult to quantify and individuals indicated that their levels of pain fluctuated many times within a single 
day ${ }^{19}$. To some extent this difficulty was addressed by the introduction of a VAS that required users to indicate their perceived level of pain or discomfort "when using their wheelchair'. These findings confirm those of Samuelsson et al ${ }^{20}$ who reported reduction in seating discomfort and back pain using VAS following wheelchair intervention.

Despite some users having a previous powered chair ${ }^{3}$, the group as a whole reported significant improvements in mobility. However, no significant improvements were noted in 'independence' or in 'social life'. It may be that changes in independence and social life need more time to be established in the lives of users, while mobility is an immediate positive benefit of provision of an EPIOC. A longer follow up period may elucidate this further. Alternatively, the users may have perceived 'independence' as activities of daily living which would not be affected by EPIOC provision.

This study supports the view that the EQ-5D could potentially be developed in this way. Further research on the use of the VASs, each representing one of the EQ-5D dimensions, is indicated.

Limitations of the study

One main limitation to this study was that the study group was heterogeneous. However the provision of an EPIOC is determined by the severity of disability rather than any specific diagnosis. It is our observation, however, that some groups of users e.g. those with Muscular Dystrophy require more complex chair prescriptions and change more rapidly than some other groups e.g. cerebrovascular disease. Larger 
Powered wheelchairs and quality of life

groups of users will need to be studied before differences between diagnostic groups can be identified.

The mean duration of time from provision of chair to assessment was only about three months. As many users require modification to their chairs, which can take time, some users may not have acquired maximum benefit from their chair at the time of follow up. A longer follow-up period to allow users to practice with their new chairs is indicated.

\section{Conclusion:}

The introduction of an EPIOC into wheelchair-users lives has not significantly altered their perceived health state. It has, however significantly improved their perception of several important aspects of their lives. These were reduced pain and discomfort, and improved levels of mobility and perceived quality of life. Extension to the EQ-5D using visual analogue scales has provided a more holistic set of outcome measures that demonstrate quality of life benefits beyond that of health state alone.

\section{Acknowledgements}

We are indebted to the EPIOC team at the Stanmore Disablement Services Centre for their advice, assistance and encouragement, particularly to Margaret White and Lorraine Dixon for administrative support.

Funding: North Thames (West) Regional Audit funds 
Powered wheelchairs and quality of life

References

1 Department of Health. Powered indoor/outdoor wheelchairs for severely disabled people. NHS executive Health Service Guidelines 1996;Health Service Guidelines

(96) $34: 1-10$

2 Ward J, Hall M, Frank A, Orwell N, Belcher M, McCullagh C. The set-up and audit of a regional EPIOC service. Posture and Mobility 1999;9:5-11.

3 Frank A, Ward J, Orwell N, McCullagh C, Belcher M. Introduction of the new NHS Electric Powered Indoor/outdoor Chair (EPIOC) service: benefits, risks and implications for prescribers. Clinical Rehabilitation 2000;14:665-673.

4 Hunter J, Muir K. Measuring the benefits of NHS Indoor/Outdoor Powerchair provision. Newsletter of the British Society of Rehabilitation Medicine $1998 ; 10($ Abstract)

5 Dyson A, Field S. Electrically powered Indoor/outdoor wheelchairs (EPIOCs): users views. South Downs Health NHS Trust, Brighton, 1999;1-23

6 Sanderson D. Evaluation of the Powered Wheelchair and Voucher Scheme Initiatives for the NHS Executive and the Department of Health. Health Economics Consortium, University of York, York, 1999;1-72 
7 Smith C, McCreadie M, Unsworth J. Prescribing wheelchairs: the opinions of wheelchair users and their carers. Clinical Rehabilitation 1995;9:74-80.

8 Kersten P, Mullee M, Smith J, McLellan L, George S. Generic health status measures are unsuitable for measuring health status in severely disabled people. Clinical Rehabilitation 1999;13:219-228.

9 Brooks R, EuroQuol Group. EuroQol: the current state of play. Health Policy 1996;37:53-72.

10 Nicholl CR, Lincoln NB, Francis VM, Stephan TF. Assessing quality of life in people with multiple sclerosis. Disability and Rehabilitation 2001; 23: 597-603

11 Wolfe F, Hawley DJ. Measurement of the quality of life in rheumatic disorders using the EuroQol. British Journal of Rheumatology 1997; 36: 786-793

12 The EuroQol Group. EuroQol EQ-5D user guide. The EuroQol Foundation, The Netherlands, 1996;1-12

13 Cowan D, Turner-Smith A. The user's perspective on the provision of electronic asstive technology: equipped for life? British Journal of Occupational Therapy 1999;62:2-6. 
Powered wheelchairs and quality of life

14 Cooper E, Fyfe N. The provision of powered wheelchairs: one year on. British Journal of Therapy and Rehabilitation 1998;5:280-281.

15 Ebrahim S. Clinical and public health perspectives and applications of healthrelated quality of life measurement. Social Science and Medicine 1995;41:1383-1394.

16 Solari A, Filippini G, Gasco P, et al. Physical rehabilitation has a positive effect on disability in multiple sclerosis patients. Neurology 1999;52:57-62.

17 Collin C. Measurement of disability and handicap. In: Greenwood R, Barnes M, McMillan T, Ward C, (eds) Neurological Rehabilitation. Edinburgh: Churchill Livingstone, 1993;137-145.

18 Frank AO, McCullagh C, Pugh M, Orwell NO, Blackman G, Belcher M. Attempted systematic annual review of a regional electrically powered indoor/outdoor chair service. Proceedings of the Recent Advances in Assistive Technology \& Engineering Conference, Birmingham 2001 commented on in Posture and Mobility 2001; 14: 20

19 De Souza L, Frank A. Subjective pain experience of people with chronic back pain. Physiotherapy Research International 2000;5:207-219.

20 Samuelsson K, Larsson H, Thyberg M, Gerdle B. Wheelchair seating intervention. Results from a client-centred approach. Disability and Rehabilitatiaon 2001; 23 (15) $677-682$ 
Powered wheelchairs and quality of life 
Table 1

Main diagnosis of the study group

\begin{tabular}{|c|c|c|}
\hline Diagnoses: & $\begin{array}{l}\text { Interviewed } \\
\text { prior to } \\
\text { receiving } \\
\text { EPIOC }(n=64)\end{array}$ & $\begin{array}{l}\text { Interviewed } \\
\text { prior to and } \\
\text { following receipt } \\
\text { of EPIOC }(n=51)\end{array}$ \\
\hline Multiple sclerosis & 10 & 9 \\
\hline Muscular dystrophy & 8 & 6 \\
\hline Other neurological & 8 & 6 \\
\hline Cerebral palsy & 8 & 5 \\
\hline Spinal cord injury & 8 & 4 \\
\hline $\begin{array}{l}\text { Cerebrovascular } \\
\text { disease }\end{array}$ & 6 & 6 \\
\hline Musculoskeletal & 5 & 5 \\
\hline Mixed disabilities & 3 & 3 \\
\hline Rheumatoid arthritis & 3 & 2 \\
\hline Polio & 2 & 2 \\
\hline Miscellaneous/other & 3 & 3 \\
\hline Age: & $\begin{array}{ll}\text { mean } & 52 \\
\text { sd } & 21 \\
\end{array}$ & $\begin{array}{ll}\text { mean } & 54 \\
\text { sd } & 21 \\
\end{array}$ \\
\hline Sex: $\quad$ Male & 28 & 23 \\
\hline
\end{tabular}


Table 2

Visual analogue scores for 49 EPIOC users before and after provision.

\begin{tabular}{|l|l|l|l|}
\hline & $\begin{array}{l}\text { Pre- } \\
\text { EPIOC }\end{array}$ & $\begin{array}{l}\text { Post- } \\
\text { EPIOC }\end{array}$ & p values \\
\hline Health & $56.9(21.9)$ & $55.7(24.2)$ & NS \\
\hline Mobility & $35.3(27.7)$ & $54.8(28.9)$ & 0.001 \\
\hline Pain/discomfort & $45.0(32.7)$ & $27.1(29.1)$ & 0.001 \\
\hline Social life & $44.4(28.9)$ & $51.8(25.6)$ & NS \\
\hline Independence & $34.3(28.9)$ & $44.0(28.1)$ & NS \\
\hline Quality of life & $53.7(27.2)$ & $63.8(26.2)$ & 0.02 \\
\hline
\end{tabular}

